\title{
POSSIBLE CHANGES IN THE CORE-MANTLE AND INNER-OUTER CORE BOUNDARIES
}

\author{
J. A. JACOBS \\ Director, Institute of Earth and Planetary Physics, University of Alberta, \\ Edmonton, Alberta, Canada
}

\begin{abstract}
This paper investigates the possibility that the boundaries between the Earth's mantle and core and between the inner and outer core might show temporal changes. The evolution of the Earth's core is not discussed, but the question is raised as to whether these boundaries might not undergo small changes both in size and shape over comparatively short time intervals.
\end{abstract}

Variations in the length of the day with time scales of a few years (the so-called 'decade' fluctuations) are usually 'explained' by the transfer of angular momentum between the mantle and liquid core. This in turn implies some form of core-mantle coupling. It is difficult, however, to make quantitative estimates of the horizontal stresses at the core-mantle boundary. Calculations indicate that neither viscous coupling nor electromagnetic coupling is sufficient to account for the decade fluctuations, although more refined theories and better values of the parameters involved may change these conclusions.

More recently Hide (1969) has suggested the possibility of a different kind of coupling - topographic coupling, as a result of irregular features at the core-mantle boundary. Analysis of travel times of compressional waves reflected at this boundary shows that any such topographic features cannot exceed a few $\mathrm{km}$ in height - this being the limit of resolution of present seismic techniques. Topographic 'bumps' on the core-mantle boundary may also be the cause of horizontal density variations responsible for regional gravity anomalies as well as interacting with motions in the Earth's core distorting the Earth's magnetic field. Hide and Malin (1970) thus argued that if both gravity and magnetic anomalies are the result of the same topographic features, it should be possible to find a statistically significant correlation between them - in fact they found, for spherical harmonic coefficients up to degree 4 , a correlation coefficient of 0.84 between large scale features of the Earth's non-dipole magnetic field (for epoch 1965) and the gravitational field, provided the magnetic field is displaced $160^{\circ}$ eastwards in longitude. The validity of the statistical tests used by Hide and Malin has been questioned independently by Khan (1971) and Lowes (1971) and rebutted by Hide and Malin (1971). It must be pointed out, however, that even if such a correlation does exist, it does not by itself prove the existence of bumps on the core-mantle boundary.

In a recent paper Higgins and Kennedy (1971) have re-estimated the melting point curve of iron against pressure, using experimental data on the melting of iron and shock wave experiments on the density of iron at high pressures. They found that the melting point gradient in the Earth's core is much less steep than earlier estimates 
- there being an increase in the melting point across the outer core of only $500^{\circ} \mathrm{C}$. Higgins and Kennedy also re-estimated the adiabatic gradient in the Earth's core. In the inner core they found the melting point and adiabatic gradients to be extremely flat. Assuming that the inner core is solid and the outer core fluid, so that the adiabatic and melting temperatures are the same at the inner-outer core boundary, they found that these temperatures differ by only $15^{\circ} \mathrm{C}$ at the centre of the Earth.

On the other hand they found a very sharp curvature in the adiabatic gradient in the outer core - much steeper than the melting point gradient - the adiabatic gradient being about $1250^{\circ} \mathrm{C}$ across the outer core, compared with $500^{\circ} \mathrm{C}$ for the melting point gradient. This is just the opposite to what has usually been supposed, and the mechanism which I put forward (1953) for the formation of a solid inner core and liquid outer core would no longer be valid. If temperatures were distributed along the adiabatic gradient throughout the outer core, it too would be solid and there would be no liquid outer core. Higgins and Kennedy thus concluded that the actual temperature gradient in the outer core is much less than the adiabatic gradient. If this is the case, it would pose a substantial inhibition to radial components of convection in the outer core and the question of the generation of the motions which drive the geomagnetic dynamo would have to be reconsidered. Since there are, in my opinion, great difficulties in accepting the adiabatic gradient of Higgins and Kennedy in the outer core, and since their adiabatic gradient is greater than the melting point gradient and mine is less, it would seem perhaps that in the outer core there is little difference between the melting point and adiabatic gradients and that in the inner core there is essentially no difference. If this is true then it is interesting to try and reconstruct the evolution of the inner-outer core regime and to investigate the stability of both coremantle and inner-outer core boundaries. Such an investigation is being carried out, taking into consideration also the fact that the core is most probably not pure iron but has in addition some lighter alloying element - probably silicon or sulfur. Both these elements would have the desired effect of decreasing the density and increasing the seismic velocity of pure iron. If the extra component is sulfur it could substantially decrease its melting temperature.

If the melting point and adiabatic gradients are virtually the same throughout the entire core, then perhaps parts of the mantle may from time to time become soluble in the outer core or parts of the outer core diffuse into the mantle - thereby giving rise to the topographic bumps of Hide on the core-mantle boundary. Equally interesting is the question of how sharp the inner-outer core boundary is - recent (unpublished) work by Davies on PKiKP phases indicates that the boundary is very sharp. However the position and shape of the boundary may change over the course of time and this may be instrumental in initiating and dictating motions in the outer core and also affecting the rotation of the Earth. Also if the topography of either boundary of the outer core has a direct influence on core motions, then changes in the polarity of the Earth's magnetic field may well be random as the shape of these boundaries (randomly) changes. Much effort has been spent in recent years on the problem of reversals of the Earth's magnetic field - but perhaps there is no problem - 
the change in polarity being merely a direct result of the changes in the fluid motions of the core due to changes in the shape and size of the boundaries.

\section{References}

Hide, R.: 1969, Nature 222, 1055.

Hide, R. and Malin, S. R. C.: 1970, Nature 225, 605.

Hide, R. and Malin, S. R. C.: 1971, Nature 230, 63.

Higgins, G. and Kennedy, G. C.: 1971, J. Geophys. Res. 76, 1870.

Jacobs, J. A.: 1953, Nature 172, 297.

Khan, M. A.: 1971, Nature 230, 57.

Lowes, F. J.: 1971, Nature 230, 61.

\section{DISCUSSION}

H. Takeuchi: Would you please give the adiabatic and melting point temperature distribution in the core as given by Higgins and Kennedy?

J. A. Jacobs: Yes. If the adiabatic gradient is steeper than the melting point gradient as illustrated, the outer core would also be solid. Higgins and Kennedy thus conclude that the actual temperature in the core is well above the adiabatic and very close to the melting temperature. I am not too happy with their estimate of the adiabatic gradient. 